\title{
2D MOF Nanoflake-Assembled Spherical Microstructures for Enhanced Supercapacitor and Electrocatalysis Performances
}

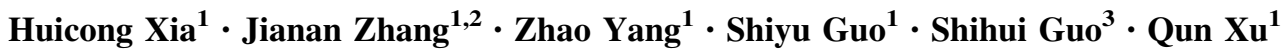

Received: 21 December 2016/Accepted: 14 February 2017/Published online: 28 March 2017

(C) The Author(s) 2017. This article is an open access publication

\section{Highlights}

- A solvothermal method was used to improve the conductivity and electrochemical activity of metal-organic framework (MOF) materials by tuning their morphology and components.

- Ni/Co-MOF nanoflakes exhibit remarkably enhanced performances including enhanced electrocatalytic performance for the oxygen reduction reaction.

- The synthetic strategy driven by rational design gives the first example of exploring MOF-derived nanomaterials to achieve improved efficiency energy storage and conversion devices.

\begin{abstract}
Metal-organic frameworks (MOFs) are of great interest as potential electrochemically active materials. However, few studies have been conducted into understanding whether control of the shape and components of MOFs can optimize their electrochemical performances due to the rational realization of their shapes. Component control of MOFs remains a significant challenge. Herein, we demonstrate a solvothermal method to realize nanostructure engineering of 2D nanoflake MOFs. The hollow structures with
\end{abstract}

Electronic supplementary material The online version of this article (doi:10.1007/s40820-017-0144-6) contains supplementary material, which is available to authorized users.

Jianan Zhang

zjn@zzu.edu.cn

$\triangle$ Qun Xu

qunxu@zzu.edu.cn

1 College of Materials Science and Engineering, Zhengzhou University, Zhengzhou 450001, People's Republic of China

2 Key Laboratory of Advanced Energy Materials Chemistry (Ministry of Education), Nankai University, Tianjin 30071, People's Republic of China

3 State Key Laboratory of Inorganic Synthesis and Preparative Chemistry, Jilin University, Changchun 130012, People's Republic of China
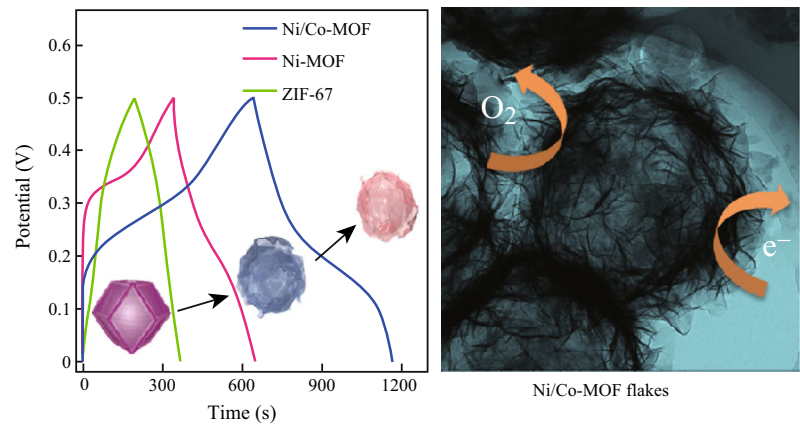

Ni/Co- and Ni-MOF (denoted as Ni/Co-MOF nanoflakes and Ni-MOF nanoflakes) were assembled for their electrochemical performance optimizations in supercapacitors and in the oxygen reduction reaction (ORR). As a result, the Ni/CoMOF nanoflakes exhibited remarkably enhanced performance with a specific capacitance of $530.4 \mathrm{~F} \mathrm{~g}^{-1}$ at $0.5 \mathrm{~A} \mathrm{~g}^{-1}$ in $1 \mathrm{M} \mathrm{LiOH}$ aqueous solution, much higher than that of $\mathrm{Ni}$ MOF (306.8 $\mathrm{F} \mathrm{g}^{-1}$ ) and ZIF-67 (168.3 $\left.\mathrm{F} \mathrm{g}^{-1}\right)$, a good rate capability, and a robust cycling performance with no capacity fading after 2000 cycles. Ni/Co-MOF nanoflakes also showed improved electrocatalytic performance for the ORR compared to Ni-MOF and ZIF-67. The present work highlights the significant role of tuning $2 \mathrm{D}$ nanoflake ensembles of $\mathrm{Ni} / \mathrm{Co}-\mathrm{MOF}$ in accelerating electron and charge transportation for optimizing energy storage and conversion devices. 
Keywords Metal-organic frameworks · Nanoflakes · Spherical microstructure - Supercapacitor .

Oxygen reduction reaction

\section{Introduction}

The design and building of metal-organic frameworks (MOFs) with controllable structures have received significant attention due to their wide range of applications such as in gas storage and separation [1-4], optoelectronics and energy storage [5-10], catalysis [11-17], and drug delivery and imaging [18-20]. With their exceptionally large surface area, abundant micropores, and variable sites for redox reactions, MOFs are considered advanced promising electrode materials for electrochemical energy storage and conversion devices such as batteries, supercapacitors, and fuel cells [8, 21-27]. The biggest problem facing individual MOFs is that they suffer from relatively low conductivity and poor electrolyte ion transport behavior, thereby restricting their efficiency for energy storage and conversion. A general approach to addressing this issue is to apply MOFs as sacrificial templates to generate porous carbon with metal or metal oxides, which can afford high conductivity and electrochemical reactivity. While high-temperature treatment inevitably results in cost increase, the intricate porous structure of MOFs cannot be employed generally [22, 28-31]. The direct application of a series of MOFs as electrode materials for supercapacitors, presented in Yaghi and co-workers' pioneering study, seems promising [32], but sluggish kinetics and poor long-term stability for electrochemical capacitors and electrochemical catalysis greatly limit their utilization.

Recent studies revealed that two-dimensional (2D) nanomaterials with short pathways for mass transport and multiple metallic ions create an opportunity for energy storage and conversion devices relative to their counterparts with other dimensionalities. The electron confinement in two dimensions of the ultrathin 2D nanomaterials renders compelling electronic properties [33-36]. Prominent examples include the MOF@graphene oxide designed for the lithium-sulfur battery that functioned as a battery separator to selectively sieve $\mathrm{Li}^{+}$ions while blocking polysulfides [9], the 2D porphyrin paddlewheel framework-3 (PPF-3) nanoflake successfully used as an electrode for a supercapacitor [37], the 2D metal oxide/hydroxide graphene nanohybrids that exhibited outstanding catalytic behavior for the oxygen reduction reaction (ORR) [38], and metal-nitrogen-containing mesoporous carbon/graphene nanoflakes exhibiting enhanced ORR performance [39]. Despite the fact that these state-of-the-art 2D nanoarchitectures show great potential in optimizing energy storage and catalysis, they usually show limited performance because of the following two issues: (I) most MOFs possess micropores with diameters less than $2 \mathrm{~nm}$, thus blocking the transport of atoms, ions, and large molecules. Therefore, the coexistence of micropore-mesoporemacropores in MOFs is highly desired; and (II) the reactivity of MOFs on the pseudocapacitance and catalytic performance should be enhanced by optimizing the valence variability of the metallic ions as redox centers. In this regard, it will be of great significance to develop MOFs with multiple structures that consist of hierarchical pores and 2D nanoflakes, and also a 3D large interconnected network that can afford efficient charge, mass exchange, and low internal resistance.

Herein, we report a facile strategy to synthesize 2D MOF nanoflake-assembled spherical microstructures composed of ultrathin $\mathrm{Ni} / \mathrm{Co}$ - and Ni-imidazolate framework nanoflakes as subunits (simplified as Ni/Co-MOFs nanoflakes). This unique superstructure with 3D accessible sites, maximized surface area, and synergistic effect of dual metallic $\left(\mathrm{Ni}^{3+} / \mathrm{Ni}^{2+}\right.$ and $\left.\mathrm{Co}^{3+} / \mathrm{Co}^{2+}\right)$ ions is highly beneficial in enhancing electrochemical storage and conversion. As a result, the Ni/Co-MOFs nanoflakes exhibit remarkable performances with a specific capacitance of $530.4 \mathrm{~F} \mathrm{~g}^{-1}$ at $0.5 \mathrm{~A} \mathrm{~g}^{-1}$ in $1 \mathrm{M} \mathrm{LiOH}$ aqueous solution, 1.72 and 3.15 times higher than that of Ni-MOF nanoflakes (306.8 $\mathrm{F} \mathrm{g}^{-1}$ ) and ZIF-67 (168.3 $\mathrm{F} \mathrm{g}^{-1}$ ), respectively; good rate capability and robust cycling performance with no capacity fading after 2000 cycles. Additionally, Ni/Co-MOF nanoflakes show higher electrocatalytic activity for the ORR than Ni-MOF nanoflakes and ZIF-67. The smart synthetic strategy employing rational design gives the first example of exploring MOF-derived nanomaterials in achieving more efficient energy storage and conversion devices.

\section{Materials and Methods}

\subsection{Chemicals}

Cobalt nitrate hexahydrate $\left(\mathrm{Co}\left(\mathrm{NO}_{3}\right)_{2} \cdot 6 \mathrm{H}_{2} \mathrm{O}\right)$ was purchased from Zhengzhou Chemical Reagents Co., Ltd. Anhydrous methanol was purchased from Tianjin Chemical Reagents Co., Ltd. 2-Methylimidazole ( $\mathrm{MW}=82.10$, 
$\mathrm{C}_{4} \mathrm{H}_{6} \mathrm{~N}_{2}$ ) was purchased from Sigma-Aldrich. Nickel(II) nitrate hexahydrate $\left(\mathrm{Ni}\left(\mathrm{NO}_{3}\right)_{2} \cdot 6 \mathrm{H}_{2} \mathrm{O}\right)$ was purchased from Sinopharm Chemical Reagent Co., Ltd. All chemicals used were of analytical grade and used without further purification.

\subsection{Synthesis of ZIF-67}

ZiF-67 was synthesized according to the previous literature [40]. A solution of 2-methylimidazole $(7.5 \mathrm{mM}, 15 \mathrm{~mL})$ in methanol was slowly added to $15 \mathrm{~mL}$ of a $\mathrm{Co}\left(\mathrm{NO}_{3}\right)_{2} \cdot 6 \mathrm{H}_{2} \mathrm{O}$ $(1.9 \mathrm{mM})$ methanol solution using a syringe at room temperature. After ultrasonic irradiation for $15 \mathrm{~min}$, the ZIF-67 nanocrystals were separated via centrifugation.

\subsection{Synthesis of Ni-MOF Nanoflakes}

Ni-MOF nanoflakes were synthesized by a solvothermal method. The as-prepared ZiF-67 nanocrystals were dispersed in $15 \mathrm{~mL}$ methanol followed by the addition of $15 \mathrm{~mL} \mathrm{Ni}\left(\mathrm{NO}_{3}\right)_{2} \cdot 6 \mathrm{H}_{2} \mathrm{O}$ methanol solution $(1.9 \mathrm{mM})$. The above mixture was transferred to a Teflon-lined stainlesssteel autoclave and kept at $120{ }^{\circ} \mathrm{C}$ for $1 \mathrm{~h}$. Finally, the product was obtained by centrifugation, washed three times with methanol, and dried at $60{ }^{\circ} \mathrm{C}$ for $12 \mathrm{~h}$.

\subsection{Synthesis of Ni/Co-MOF Nanoflakes}

As-prepared $\mathrm{ZiF}-67$ nanocrystals were dispersed in $15 \mathrm{~mL}$ methanol followed by the addition of $15 \mathrm{~mL}$ methanol containing $\mathrm{Ni}\left(\mathrm{NO}_{3}\right)_{2} \cdot 6 \mathrm{H}_{2} \mathrm{O}(0.95 \mathrm{mM})$ and $\mathrm{Co}\left(\mathrm{NO}_{3}\right)_{2} \cdot 6 \mathrm{H}_{2} \mathrm{O}(0.95 \mathrm{mM})$. The product was obtained by centrifugation, washed three times with methanol, and dried at $60{ }^{\circ} \mathrm{C}$ for $12 \mathrm{~h}$.

\subsection{Characterization}

The morphologies of the samples were studied by fieldemission scanning electron microscopy (FE-SEM, JEORJSM-6700F) and transmission electron microscopy (TEM, FEI Tecnai G2 20) with an accelerating voltage of $200 \mathrm{kV}$. Powder XRD patterns were collected using a Y-2000 X-ray diffractometer with copper $K_{\alpha}$ radiation $(\lambda=1.5406 \AA)$ at $40 \mathrm{kV}$ and $40 \mathrm{~mA}$. Fourier transform infrared (FTIR) spectra of the products were recorded on a TENSOR 27 FTIR spectrometer (Bruker) in the absorption mode with a resolution of $2 \mathrm{~cm}^{-1}$. The $\mathrm{X}$-ray photoelectron spectroscopy (XPS) measurements were performed with an ESCA LAB 250 spectrometer using a focused monochromatic $\mathrm{Al}-K_{\alpha}$ line $(1486.6 \mathrm{eV}) \mathrm{X}$-ray beam with a diameter of $200 \mu \mathrm{m}$. Thermal gravimetric analysis (TGA) was conducted on an SMP/PF7548/MET/600 W instrument from 50 to $800{ }^{\circ} \mathrm{C}$ with a heating rate of $10{ }^{\circ} \mathrm{C} \mathrm{min}^{-1}$ in a nitrogen atmosphere.

\subsection{Electrochemical Measurements}

\subsubsection{Supercapacitor Measurements (Three-Electrode System)}

The capacitance performances of the samples were evaluated with a three-electrode system on an electrochemical workstation (CHI 760E, CH Instrument, China) at room temperature. The suspension with active materials at a concentration of $1.0 \mathrm{mg} \mathrm{mL}^{-1}$ was prepared by sonicating $1 \mathrm{mg}$ of active materials in $1 \mathrm{~mL}$ ethanol containing Nafion (Sigma-Aldrich, $5 \mathrm{wt} \%$ ) at a volume ratio of 995:5. Then, $20 \mu \mathrm{L}$ of ink was dropped onto a glassy carbon disk (diameter $5 \mathrm{~mm}$ ) and dried thoroughly in air, resulting in a catalyst loading of $0.1 \mathrm{mg} \mathrm{cm}^{-2}$. The auxiliary and reference electrodes were $\mathrm{Pt}$ wire and $\mathrm{Ag} / \mathrm{AgCl}$, respectively. The electrochemical measurements were carried out in $1 \mathrm{M}$ $\mathrm{LiOH}$ solution.

The potential range for cyclic voltammetry (CV) tests was $0-0.5 \mathrm{~V}$, and the scan rates were $5,10,25,50,75,100$, 150 , and $200 \mathrm{mV} \mathrm{s}^{-1}$. Galvanostatic charge-discharge (GCD) measurements were done from 0 to $0.5 \mathrm{~V}$ at different current densities of $0.5,1,2,4,6,8$, and $10 \mathrm{~A} \mathrm{~g}^{-1}$. The gravimetric specific capacitance $C\left(\mathrm{~F} \mathrm{~g}^{-1}\right)$ based on the discharge curves was calculated by:

$C=(I \times \Delta t) /(\Delta V \times m)$

where $I$ is the discharge current (A), $\Delta t$ is the discharge time (s), $\Delta V$ is the potential window $(\mathrm{V})$, and $m$ is the mass of active materials on the test electrode $(\mathrm{g})$.

\subsubsection{Oxygen Reduction Reaction (ORR)}

To prepare the working electrode, $5 \mathrm{mg}$ of catalyst and $5 \mathrm{mg}$ carbon black (Alfa Aesar, 99.9+ wt\%) were dispersed in a mixture of $950 \mu \mathrm{L}$ ethanol and $50 \mu \mathrm{L}$ Nafion (Sigma-Aldrich, $5 \mathrm{wt} \%$ ) under sonication for $30 \mathrm{~min}$ to obtain a homogeneous slurry. Then, $8 \mu \mathrm{L}$ of this catalyst ink was loaded onto a glassy carbon rotating disk electrode of diameter $5 \mathrm{~mm}$, resulting in the catalyst loading of $0.2 \mathrm{mg} \mathrm{cm}^{-2}$. The electrode was dried under dissolvent conditions for $5 \mathrm{~h}$.

Electrochemical impedance spectral measurements were carried out in the frequency range from $100 \mathrm{kHz}$ to $10 \mathrm{mHz}$ on a $\mathrm{CHI} 760 \mathrm{E}$ electrochemical workstation. Cyclic voltammetry (CV) and rotating disk electrode (RDE) measurements (Pine Research Instruments, USA) were conducted using a standard three-electrode system. The catalyst-coated glassy carbon electrode, an $\mathrm{Ag} / \mathrm{AgCl}$ 
electrode in saturated $\mathrm{KCl}$ solution, and $\mathrm{Pt}$ wire were used as the working, reference, and counter electrodes, respectively. The electrolyte was $0.1 \mathrm{M}$ potassium hydroxide $(\mathrm{KOH})$ aqueous solution. The potential measured against the $\mathrm{Ag} / \mathrm{AgCl}$ electrode was converted to the potential versus the reversible hydrogen electrode (RHE) according to $E($ vs. $\mathrm{RHE})=E($ vs. $\mathrm{Ag} / \mathrm{AgCl})+0.197+0.059 \mathrm{pH}$. All measurements were carried out at room temperature.

For the ORR at an RDE, the working electrode was scanned cathodically at a rate of $10 \mathrm{mV} \mathrm{s}^{-1}$ at different rotating speeds from 400 to $2500 \mathrm{rpm}$ in $\mathrm{O}_{2}$-saturated $0.1 \mathrm{M} \mathrm{KOH}$ aqueous solution. Koutecky-Levich (K-L) plots were analyzed at various electrode potentials. The slopes of their linear fit lines were used to calculate the electron transfer number (n) on the basis of the $K-L$ equation:

$\frac{1}{J}=\frac{1}{J_{\mathrm{L}}}+\frac{1}{J_{\mathrm{K}}}=\frac{1}{B \omega^{0.5}}+\frac{1}{J_{\mathrm{K}}}$

$J_{\mathrm{L}}=0.62 n F C_{0} D_{0}^{2 / 3} \omega^{0.5} v^{-1 / 6}$

$B=0.62 n F C_{0} D_{0}^{2 / 3} v^{-1 / 6}$

$J_{\mathrm{K}}=n F k C_{0}$

where $J$ is the measured current density, $J_{\mathrm{L}}$ and $J_{\mathrm{K}}$ are the diffusion- and kinetic-limited current densities, $\omega$ is the rotation speed $\left(\mathrm{rad} \mathrm{s}^{-1}\right), n$ is the transferred electron number, $F$ is the Faraday constant $\left(96,485 \mathrm{C} \mathrm{mol}^{-1}\right), C_{0}$ is the $\mathrm{O}_{2}$ concentration in the electrolyte $\left(1.26 \times 10^{-6}\right.$ mol cm$\left.~^{-3}\right), D_{0}$ is the diffusion coefficient of $\mathrm{O}_{2}$ in the electrolyte $\left(1.93 \times 10^{-5} \mathrm{~cm}^{2} \mathrm{~s}^{-1}\right)$, and $v$ is the kinetic viscosity $\left(0.01009 \mathrm{~cm}^{2} \mathrm{~s}^{-1}\right)$.

\section{Results and Discussion}

Scheme 1 illustrates the wet-chemical protocol for synthesizing the 2D Ni/Co-MOF nanoflake-assembled superstructure via morphology transformation of ZIF$67\left(\left[\mathrm{Co}(\mathrm{MeIm})_{2}\right]_{\mathrm{n}}\right)(\mathrm{MeIm}=$ methylimidazole $)$. Typically,

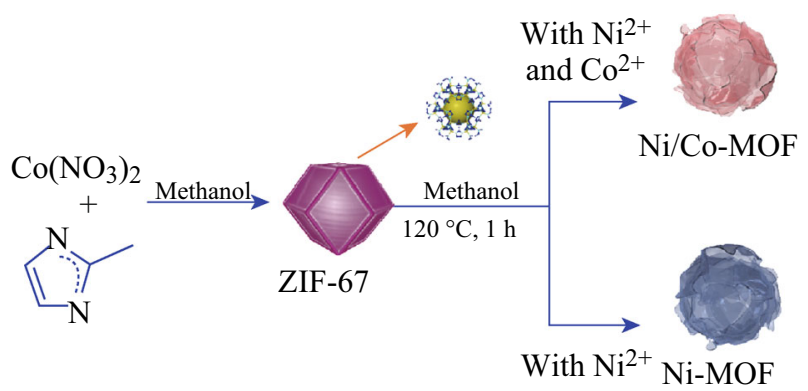

Scheme 1 Schematic illustration of the synthesis of Ni/Co-MOF nanoflakes and Ni-MOF nanoflakes
ZIF-67 rhombododecahedron nanoseeds with sizes of around $400 \mathrm{~nm}$ were prepared employing $\mathrm{Co}\left(\mathrm{NO}_{3}\right)_{2}$ as metallic source, 2-methylimidazole as organic linker, and methanol as solvent (Fig. S1a). Sequentially, ZIF-67 rhombododecahedron nanoseeds were dispersed in methanol followed by the addition of solutions of $\mathrm{Ni}\left(\mathrm{NO}_{3}\right)_{2}$ and $\mathrm{Co}\left(\mathrm{NO}_{3}\right)_{2}$ in methanol. The parent ZIF-67 would readily evolve into a distinctive hollow nanocage after $60 \mathrm{~min}$ of solvothermal treatment in methanol. In the initial stage, we suggest that $\mathrm{Ni}^{2+}$ partly substituted $\mathrm{Co}^{2+}$ in the framework, which could retain the ZIF-67 crystalline lattice. Over time, the ZIF-67 rhombododecahedron were gradually etched and the Ni/Co-MOF nanoflakes were simultaneously formed and covered the surface of the polyhedron, which was accompanied by a size increase in the inner core from 400 to $500-700 \mathrm{~nm}$. It was observed that all of the solid ZIF-67 had transformed to 2D hollow MOF nanoflake spherical microstructures.

As demonstrated in the TEM (Fig. S1b, c) and SEM (Fig. 1a) images, both Ni/Co-MOF nanoflakes and NiMOF nanoflakes show distinctive hollow nanocages assembled by overlapping nanoflakes and hollow superstructures are well-defined with the average diameter of $500-700 \mathrm{~nm}$ corresponding to the ZIF-67 precursor ( $400 \mathrm{~nm}$ ). Taking a closer look at the detailed structure, each spherical microstructure is essentially a 3D structure comprised of numerous building blocks of $2 \mathrm{D}$ ultrathin nanoflakes (Fig. 1b, c). To further figure out the detailed structure of the Ni/Co-MOF nanoflakes, magnified TEM images and XRD patterns of Ni/Co-MOF nanoflakes were collected (Fig. 1d, e). It was found that an individual nanosheet of $\mathrm{Ni} / \mathrm{Co}-\mathrm{MOF}$ nanoflakes consists of numerous white lines with widths of $<1 \mathrm{~nm}$ implying the microporous structure of the Ni/Co-MOF nanosheets, which greatly benefits mass transport during electrochemical reactions. The XRD patterns (Fig. 1e) of the Ni/Co-MOF nanoflakes, Ni-MOF nanoflakes, and ZIF-67 were measured. The peaks of the parent ZIF-67 seeds matched the patterns of simulated ZIF-67, whereas the characteristic peaks of ZIF-67 $\left(12.76^{\circ}\right.$ and $\left.18.11^{\circ}\right)$ disappeared and two broad peaks emerged at $10.48^{\circ}$ and $33.96^{\circ}$ when $\mathrm{Ni} / \mathrm{Co}-$ MOF nanoflakes and Ni-MOF nanoflakes formed. It was proposed that the coordination mode between $\mathrm{Ni}^{2+}$ and $\mathrm{Co}^{2+}$ ions and 2-methylimidazole was influenced by the involvement of methanol molecules in the unit cell [41]. Such influences result in structural evolution from a rhombododecahedron to the 2D MOF nanoflake-assembled spherical microstructure. The investigation of the valence states of Ni and Co was carried out using XPS (Fig. 2a, b, S2). The characteristic bands at 873.3 and $796.9 \mathrm{eV}$ are the fingerprints of $\mathrm{Ni}^{2+}$ and $\mathrm{Co}^{2+}$ in tetrahedral coordination, implying the persistence of the coordination mode during the phase transformation [42-45]. The Ni $2 p$ spectrum of 

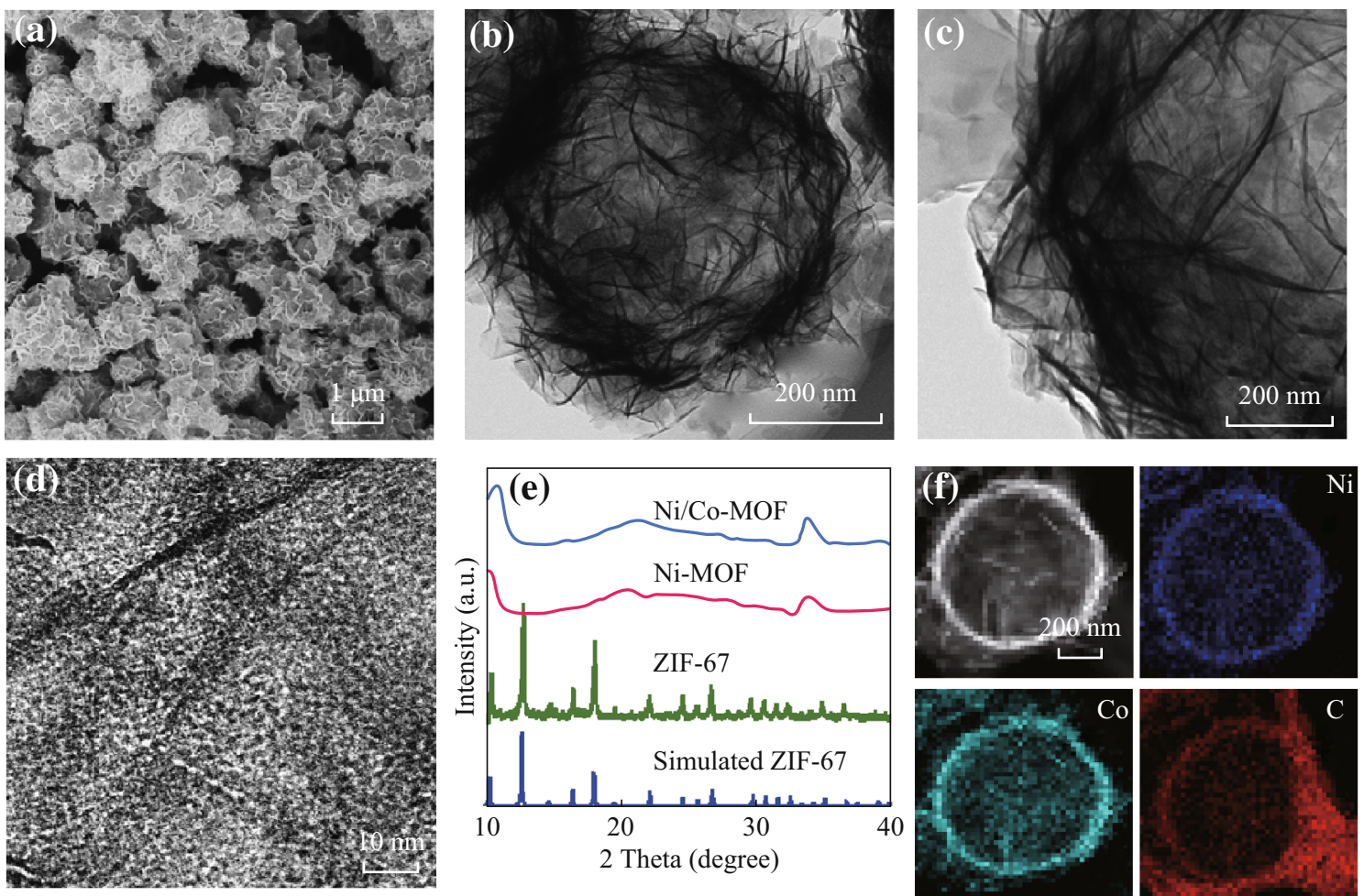

Fig. 1 a SEM images of Ni/Co-MOF nanoflakes. b, $\mathbf{c}$ TEM images of Ni/Co-MOF nanoflakes. $\mathbf{d}$ Large-magnified TEM and SAED patterns of $\mathrm{Ni} / \mathrm{Co}-\mathrm{MOF}$ nanoflakes. e XRD patterns of Ni/Co-MOF nanoflakes, Ni-MOF nanoflakes, and ZIF-67. f STEM-energy-dispersive spectroscopy (EDS) mapping of Ni/Co-MOF nanoflakes
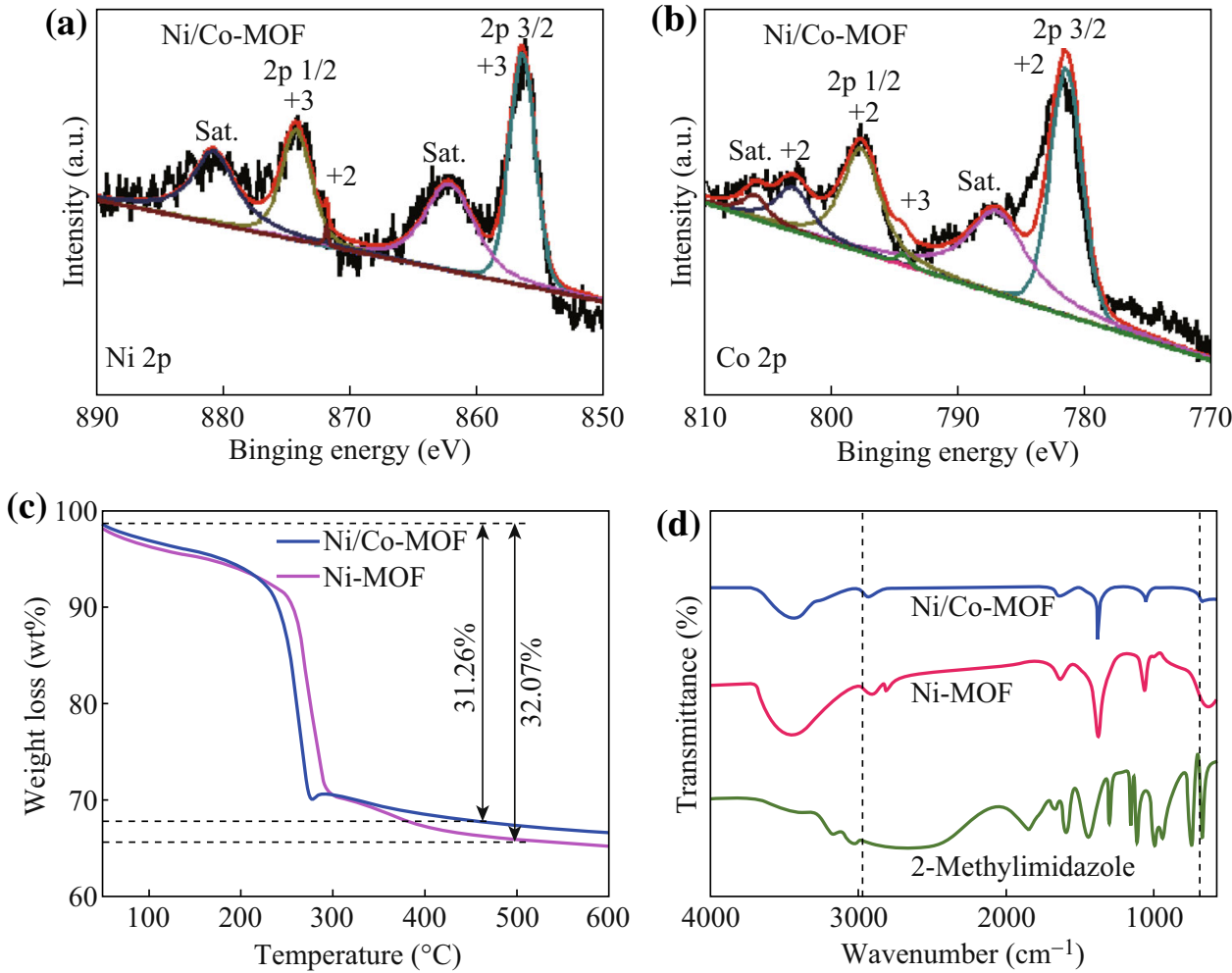

Fig. 2 a Ni $2 p$ XPS spectra and b Co $2 p$ spectra of Ni/Co-MOF nanoflakes. $\mathbf{c}$ TGA of Ni/Co-MOF nanoflakes and Ni-MOF nanoflakes. d FTIR spectra of Ni/Co-MOF nanoflakes, Ni-MOF nanoflakes, and 2-methylimidazole 
the Ni/Co-MOF can be deconvolved into two spin-orbit doublets. The first doublet at $856.6 \mathrm{eV}$ and the second at $874.3 \mathrm{eV}$ could be assigned to $\mathrm{Ni}^{3+}$ [46]. The $\mathrm{Ni}^{3+} / \mathrm{Ni}^{2+}$ ratio is $\sim 1.22$ for $\mathrm{Ni} / \mathrm{Co}-\mathrm{MOF}$. The binding energy at $781.6 \mathrm{eV}$ corresponds to the spin-orbit characteristic of $\mathrm{Co}^{2+}$. The spectral $\mathrm{Co}^{3+} / \mathrm{Co}^{2+}$ ratio obtained from their respective main lines is $\sim 0.78$ for the Ni/Co-MOF. The elemental distributions of $\mathrm{Ni} / \mathrm{Co}-\mathrm{MOF}$ and $\mathrm{Ni}-\mathrm{MOF}$ nanoflakes were further confirmed from STEM-energydispersive spectroscopy (EDS) mapping (Fig. S2). As shown in Fig. If and Fig. S2, Co, Ni, C, and $\mathrm{N}$ were uniformly distributed on the overall nanoflakes, and the $\mathrm{Ni}$ and Co elements were in the loaded Ni/Co-MOF nanoflakes.

The TGA data of Ni/Co-MOF and Ni-MOF nanoflakes were collected under air at the heating rate of $10{ }^{\circ} \mathrm{C} \mathrm{min}{ }^{-1}$ from room temperature to $800{ }^{\circ} \mathrm{C}$. The slight weight loss ( $<8 \mathrm{wt} \%$ ) before $220{ }^{\circ} \mathrm{C}$ is attributed to water that is bonded to the imidazole group of the MOF framework via $\mathrm{H}$-bonds. The typical weight loss of Ni/Co-MOF and NiMOF nanoflakes calculated from the TGA data (Fig. 2c) was determined to be 31.26 and $32.07 \mathrm{wt} \%$ at 250 and
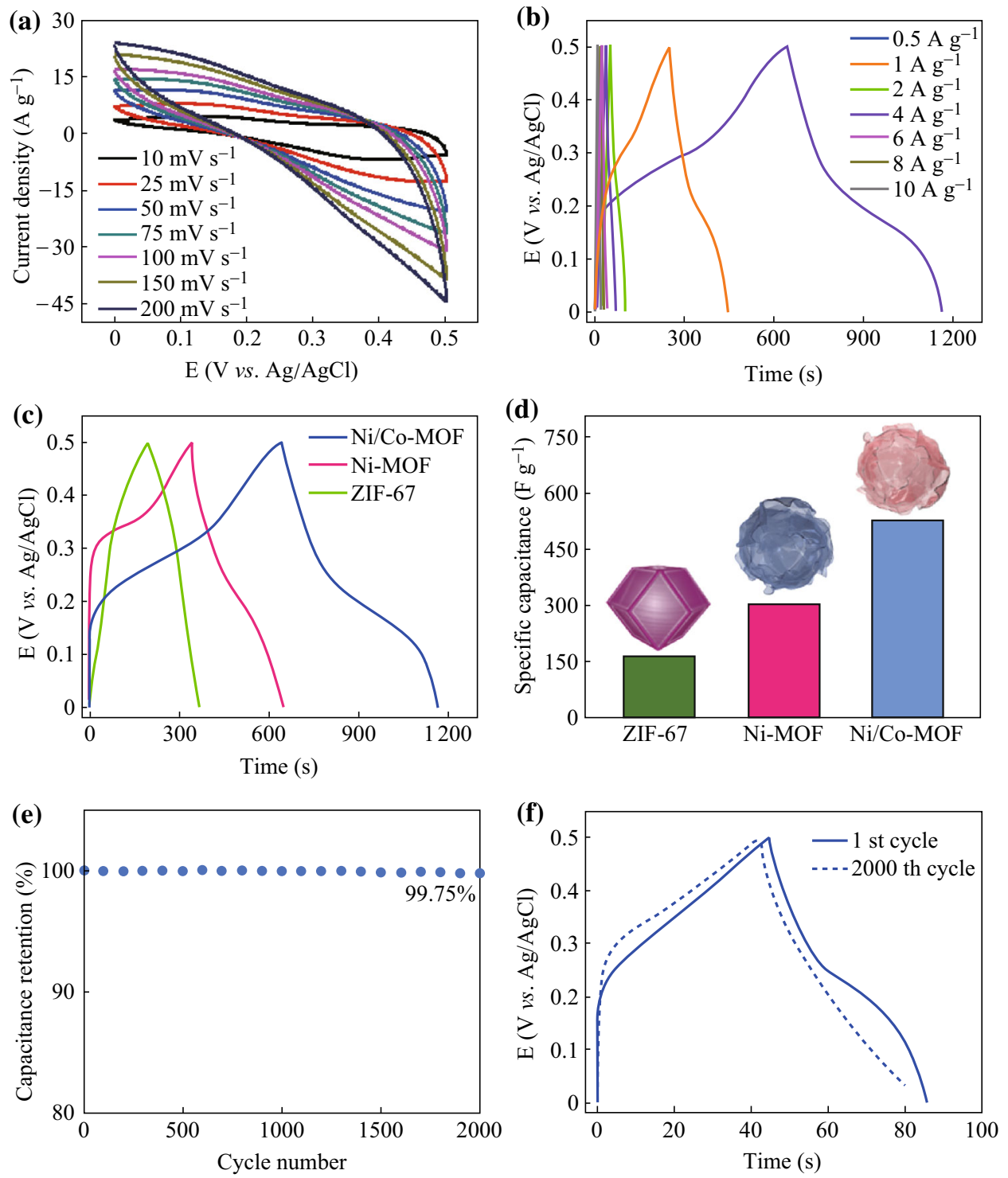

Fig. 3 a Cyclic voltammograms of as-prepared Ni/Co-MOF nanoflake electrodes at different scan rates in $1 \mathrm{M}$ LiOH solution. b Galvanostatic charge-discharge curves of Ni/Co-MOF nanoflakes at various currents in $1 \mathrm{M} \mathrm{LiOH}$ solution. c Galvanostatic charge-discharge curves comparison of Ni/Co-MOF nanoflakes, ZIF-67, and Ni/Co-MOF nanoflakes at various current densities in $1 \mathrm{M} \mathrm{LiOH}$ solution. d Specific capacitances of Ni/Co-MOF nanoflakes, Ni-MOF nanoflakes, and ZIF-67 electrodes at $0.5 \mathrm{~A} \mathrm{~g}^{-1}$. e Cycling performance of Ni/Co-MOF nanoflakes at a current density of $2 \mathrm{~A} \mathrm{~g}^{-1}$. f Galvanostatic charge-discharge curves of Ni/Co-MOF nanoflake supercapacitor before and after 2000 cycles measured at $2 \mathrm{~A} \mathrm{~g}^{-1}$ 
$220{ }^{\circ} \mathrm{C}$, respectively, attributed to the removal of the organic ligands. FTIR spectra of Ni/Co-MOF and Ni-MOF nanoflakes were measured to identify the surface functional groups and observe the formation of coordinated polymers. As shown in Fig. 2d, the FTIR spectrum has been studied extensively and every absorption peak was assigned to its corresponding vibration. The characterization peaks at 2923 and $584 \mathrm{~cm}^{-1}$ were attributed to the aliphatic $\mathrm{C}-\mathrm{H}$ stretch and the $\mathrm{C}=\mathrm{N}$ stretching vibrations of 2-methylimidazole. Therefore, the stretching of aliphatic $\mathrm{C}-\mathrm{H}$ and vibration of $\mathrm{C}=\mathrm{N}$ in 2-methylimidazole at 624 and $3100 \mathrm{~cm}^{-1}$ shifted to 584 and $2923 \mathrm{~cm}^{-1}$ after assembling $\mathrm{Ni} / \mathrm{Co}-\mathrm{MOF}$ or $\mathrm{Ni}-\mathrm{MOF}$, indicating the strong interaction of $\mathrm{C}=\mathrm{N}$ and $\mathrm{C}-\mathrm{H}$ groups in 2-methylimidazole with the $\mathrm{Ni}^{2+}$ or $\mathrm{Co}^{2+}$ ions [47]. Based on the above analysis, the linker between the $\mathrm{Ni}^{2+}$ and $\mathrm{Co}^{2+}$ and 2-methylimidazole remained unchanged during phase transformation.

The unique structure of the Ni/Co-MOF nanoflakes and $\mathrm{Ni}-\mathrm{MOF}$ nanoflakes inspired us to evaluate their electrochemical performance for a supercapacitor with a threeelectrode system in $1 \mathrm{M} \mathrm{LiOH}$ aqueous electrolyte. As shown in Fig. 3a and Fig. S3, CV curves of Ni/Co-MOF nanoflakes present roughly rectangular shapes and reversible bumps, suggesting the combination of electric doublelayer capacitance (EDLC) and a pseudocapacitive reaction. It should be noted that the roughly rectangular shape of the
$\mathrm{Ni} / \mathrm{Co}-\mathrm{MOF}$ nanoflakes was maintained even at a high potential scan rate of $200 \mathrm{mV} \mathrm{s}^{-1}$, indicating pure EDLC behavior and rapid formation of the double layer even at high rates. The appearance of nearly symmetric galvanostatic charge-discharge curves in Fig. $3 b$ indicates that the unique electrode has a low polarization and high chargedischarge columbic efficiency. Significantly, Fig. 3c, d shows that the Ni/Co-MOF nanoflakes exhibit a high specific capacitance of $530.4 \mathrm{~F} \mathrm{~g}^{-1}$ at $0.5 \mathrm{~A} \mathrm{~g}^{-1}, 3.15$ and 1.72 times higher than those of the Ni-MOF nanoflakes (306.8 $\left.\mathrm{F} \mathrm{g}^{-1}\right)$ and ZIF-67 $\left(168.3 \mathrm{~F} \mathrm{~g}^{-1}\right)$. As shown in Table S1, the Ni/Co-MOF nanoflakes showed a much higher specific capacitance than many previously reported MOF-based materials such as worm-like Co-MOF [23], curled sheet-like N-doped Zn-MOF [48], sodalite-structured ZIF-67 [49], and honeycomb-like Co-MOF [50]. Figure $3 \mathrm{e}$, f presents the cycling performance of the $\mathrm{Ni} / \mathrm{Co}-$ MOF nanoflakes at a current density of $2 \mathrm{~A} \mathrm{~g}^{-1}$. The Ni/ Co-MOF nanoflakes show a very stable capacitance ( $99.75 \%$ of the original capacitance) after 2000 cycles of charging and discharging, indicating long-term electrochemical stability. This is further confirmed by the inconspicuous change between the charging-discharging curves of the first and 2000th cycles. Considering their high specific capacitance and robust electrochemical stability, the Ni/Co-MOF nanoflakes present promise as an advanced
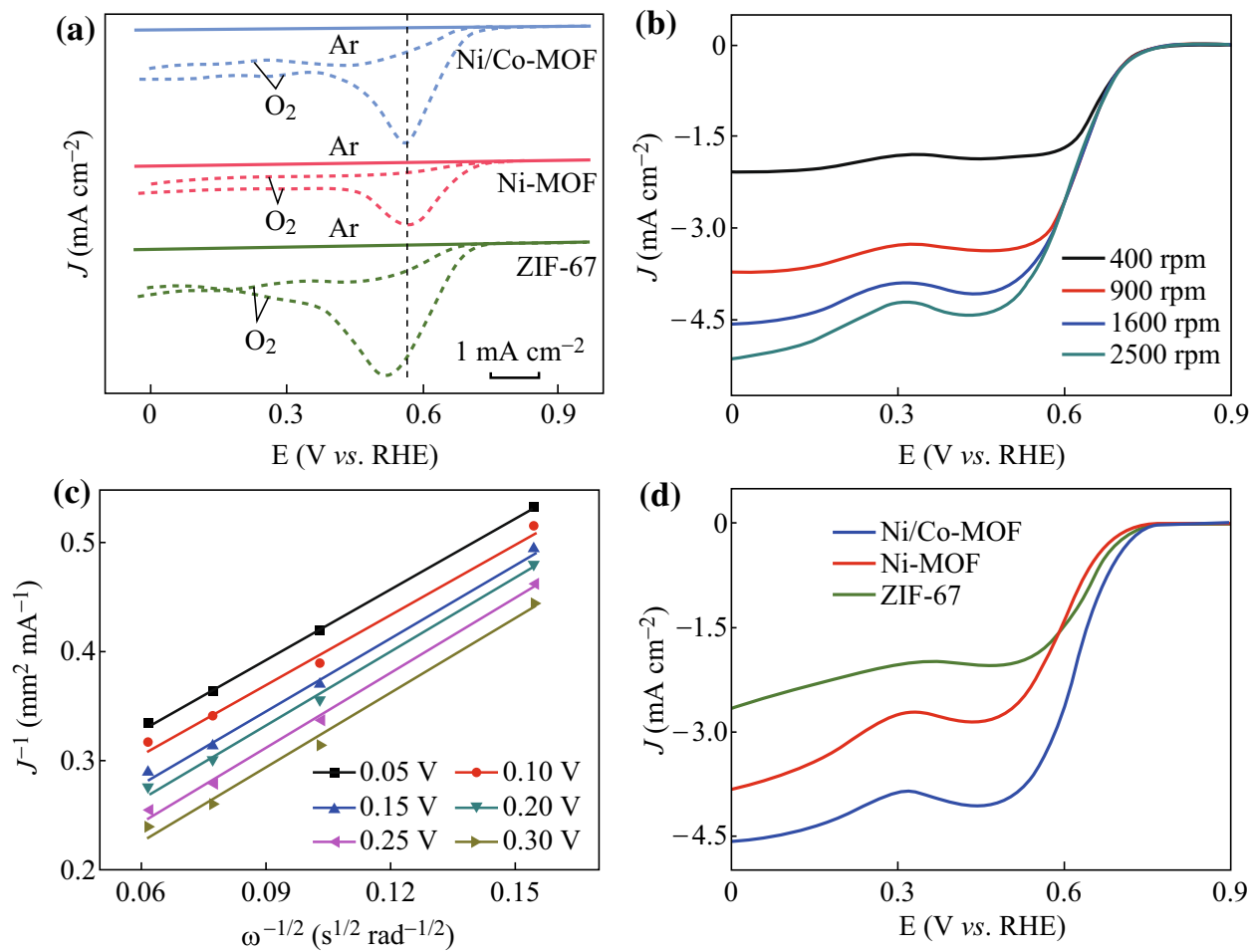

Fig. 4 a $\mathrm{CV}$ curves in Ar-saturated (solid curves) and $\mathrm{O}_{2}$-saturated (dashed curves) solutions with a sweep rate of $50 \mathrm{mV} \mathrm{s}^{-1}$. b $\mathrm{RDE}$ polarization curves of $\mathrm{Ni} / \mathrm{Co}-\mathrm{MOF}$ nanoflakes at different rotation speeds. Scan rate: $10 \mathrm{mV} \mathrm{s}{ }^{-1}$. $\mathbf{c}$ Koutecky-Levich plots of Ni/Co-MOF nanoflakes at various potentials. d RDE polarization curves of samples at a rotation rate of $1600 \mathrm{rpm}$. Scan rate: $10 \mathrm{mV} \mathrm{s}^{-1}$ 
electrode candidate for supercapacitors. Obviously, the $\mathrm{Ni} /$ Co-MOF and Ni-MOF nanoflakes exhibit better supercapacitor performance than ZIF-67, revealing the vital role of the micropore-macropore coexisting in 2D-3D MOF nanoflakes for enhancing the energy storage. According to the property of the $\mathrm{CV}$ and GCD curves of Ni/Co-MOF (potential window, oxide reduction peak, and pseudocapacitive behavior), possible active sites are the $\mathrm{Co}^{3+} / \mathrm{Co}^{2+}$ and $\mathrm{Ni}^{3+} / \mathrm{Ni}^{2+}$ ions in the structure. As shown in Fig. S4, the Ni/Co-MOF nanoflakes show terrible capacitance (70.85\% of the original capacitance) after 3000 cycles of charging and discharging, which is probably due to destruction of the morphology.

The as-prepared Ni/Co-MOF nanoflakes also work well as advanced non-metal catalysts for enhancing the ORR. Figure $4 \mathrm{a}$ shows the $\mathrm{CVs}$ of $\mathrm{Ni} / \mathrm{Co}-\mathrm{MOF}$ nanoflakes, NiMOF nanoflakes, and ZIF-67 performed in Ar- and $\mathrm{O}_{2-}$ saturated $0.1 \mathrm{M} \mathrm{KOH}$ solutions. The auxiliary and reference electrodes were $\mathrm{Pt}$ wire and $\mathrm{Ag} / \mathrm{AgCl}$, respectively. Compared with the featureless character in the Ar-saturated solutions, catalytic peaks were found in the $\mathrm{O}_{2}$-saturated solutions. A well-defined oxygen reduction cathodic peak at about $0.57 \mathrm{~V}$ can be clearly observed for the $\mathrm{Ni} / \mathrm{Co}-$ MOF nanoflakes in the $\mathrm{O}_{2}$-saturated solution, which is positively shifted about 20 and $50 \mathrm{mV}$ compared to the $\mathrm{Ni}$ MOF nanoflakes $(0.55 \mathrm{~V})$ and ZIF-67 $(0.52 \mathrm{~V})$, respectively, indicating the best effective electrochemical reduction of oxygen for the $\mathrm{Ni} / \mathrm{Co}-\mathrm{MOF}$ nanoflakes. As shown in Fig. 4b, to gain the dynamic information about the ORR, the electrocatalytic abilities were evaluated from linear sweep voltammetry (LSV) experiments carried out on a rotating disk electrode (RDE) in $\mathrm{O}_{2}$-saturated $0.1 \mathrm{M}$ $\mathrm{KOH}$ solutions at a scan rate of $10 \mathrm{mV} \mathrm{s}^{-1}$. The onset potential of Ni/Co-MOF nanoflakes for the ORR was at $\sim 0.76 \mathrm{~V}$. As shown in Fig. 4c, the corresponding $\mathrm{K}-\mathrm{L}$ plots at various electrode potentials exhibit good linearity, indicating first-order reaction kinetics toward the ORR and a similar electron transmission number for the ORR at different potentials. According to the $\mathrm{K}-\mathrm{L}$ equation, the transferred electron number in the $\mathrm{O}_{2}$ reduction process is calculated to be about 3.7 over the potential range $\sim 0.05$ to $\sim 0.3 \mathrm{~V}$; a four-electron process is the dominant pathway for the ORR at the Ni/Co-MOF nanoflake electrode. As a comparison, the ORR electrocatalytic activities of ZIF-67 and Ni-MOF nanoflakes were also investigated under the same conditions. Figure $4 \mathrm{~d}$ shows the LSV curves of ZIF-67, Ni/Co-MOF nanoflakes, and Ni-MOF nanoflakes at a rotation rate of $1600 \mathrm{rpm}$. The highest onset potential and the largest cathodic current density were observed on the Ni/Co-MOF nanoflakes. As shown in Fig. S6a, b, ZIF-67 and Ni-MOF nanoflakes typically show a two-electron-dominated transfer pathway for the ORR at lower current densities, and this is proved by the lower average numbers of transferred electrons of 2.6 and 2.8 over the potential range $\sim 0.05$ to $\sim 0.3 \mathrm{~V}$, respectively, calculated from the slopes of $\mathrm{K}-\mathrm{L}$ plots shown in Fig. S6c, d. Undoubtedly, Ni/Co-MOF nanoflakes possesses better electrocatalytic activity toward the ORR than Ni-MOF nanoflakes and ZIF-67 in the alkaline system. As confirmed from the Co $2 p$ and Ni $2 p$ XPS spectra, the dominant form of $\mathrm{Co}$ is $\mathrm{Co}^{2+}$, while that of $\mathrm{Ni}$ is $\mathrm{Ni}^{3+}$. Previous studies revealed that transition metals with mixed valences could provide donor-acceptor chemisorption sites for the reversible adsorption of oxygen and realize high electric conductivity for electron hopping between cations with different valences [46, 51], resulting in improved ORR electrocatalysis performance.

The resistance to methanol crossover effects and durability are key factors for the practical application of the catalyst in fuel cells. As shown in Fig. 5a, the chronoamperometric responses of the Ni/Co-MOF nanoflakes upon addition of $3 \mathrm{M}$ methanol were studied. With the addition of methanol, the superior stability of the $\mathrm{Ni} / \mathrm{Co}-\mathrm{MOF}$ nanoflakes for the ORR can be demonstrated from their
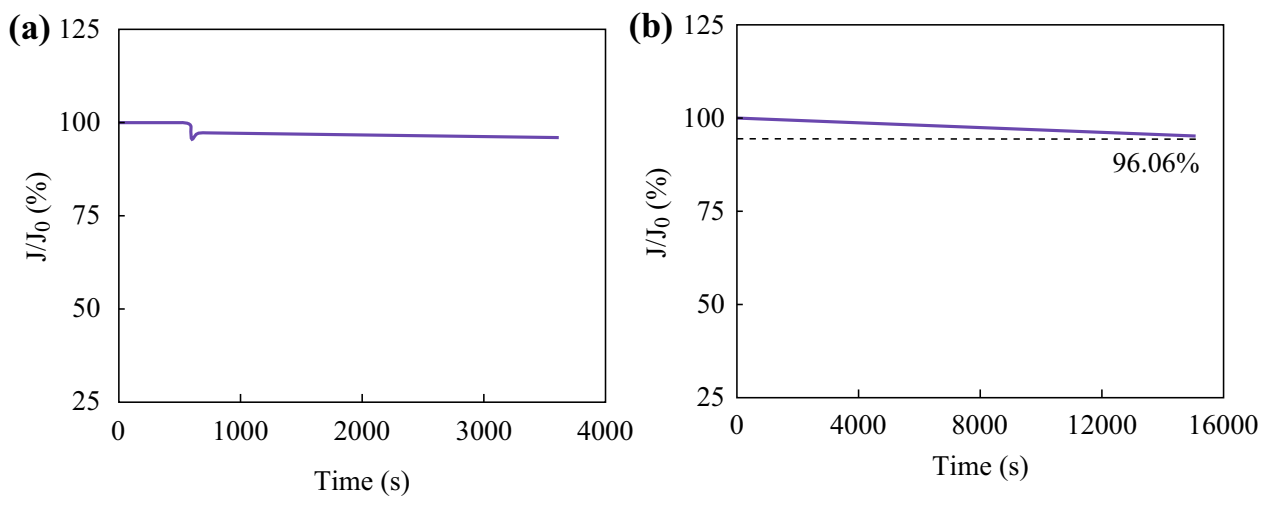

Fig. 5 a Chronoamperometric responses of Ni/Co-MOF nanoflakes in $\mathrm{O}_{2}$-saturated $0.1 \mathrm{M} \mathrm{KOH}$ solution (1600 rpm) without and with $3 \mathrm{M}$ methanol (added at $600 \mathrm{~s}$ ). b Stability evaluation at $-0.1 \mathrm{~V}$ and a rotation speed of $1600 \mathrm{rpm}$ 
negligible current decay, indicating superior tolerance against methanol crossover. In the catalytic activity durability test (Fig. 5b), Ni/Co-MOF nanoflakes exhibited a very low attenuation of $3.94 \%$ current loss at $-0.1 \mathrm{~V}$ after $15,000 \mathrm{~s}$, suggesting high stability of the Ni/Co-MOF nanoflakes. Therefore, the Ni/Co-MOF nanoflakes show promise for the ORR due to high catalytic activity, stability, and selectivity.

The results from the electrochemical performances for the supercapacitor and ORR studies indicate that the unique structure of the Ni/Co-MOF nanoflakes plays a role in optimizing the electrochemical performance of MOF materials. Significantly, the spherical microstructure assembled by interconnected MOF nanoflakes networks offers a continuous pathway for mass transportation, and the hollow nanocage formed in the textures may act as an ion-buffering reservoir for promoting transport of the electrolyte ion and oxygen, which bring more effective transmission (compared to the microporous only ZIF-67) leading to a high electrochemical performance for supercapacitors and the ORR. The synergistic effect of dual metallic $\left(\mathrm{Ni}^{3+} / \mathrm{Ni}^{2+}\right.$ and $\left.\mathrm{Co}^{3+} / \mathrm{Co}^{2+}\right)$ ions in $\mathrm{Ni} / \mathrm{Co}-\mathrm{MOF}$ nanoflakes raised the reaction activity during the energy storage and conversion, thereby optimizing electron and charge transportation and accelerating the reaction kinetics (enhancing the activity). The enhanced conductivity of $\mathrm{Ni} /$ Co-MOF can be further demonstrated by the Nyquist plots. As shown in Fig. S5, Ni/Co-MOF exhibited a lower resistance $(4.0 \Omega)$ than ZIF-67 $(8.5 \Omega)$ and Ni-MOF $(6.2 \Omega)$, suggesting a higher conductivity of the $\mathrm{Ni} / \mathrm{Co}$ MOF that is probably attributed to electron hopping between cations with different valences [46, 51]. Moreover, the spherical microstructure of Ni/Co-MOF nanoflakes provides favorable layer-by-layer assembled nanoflakes, which effectively prevent the collapse of the nanoflakes (enhancing the stability). Additionally, to investigate the cation substitution effect, we replaced $\mathrm{Ni}^{2+}$ with $\mathrm{Fe}^{2+}$ ions in the structure; solid spheres were obtained instead of the nanosheet morphology, and they showed poor electrochemical performance (not shown here). Therefore, the choice of the $\mathrm{Ni}^{3+} / \mathrm{Co}^{2+}$ couple may be suitable for constructing a high-efficiency electrode. Accordingly, the Ni/Co-MOF nanoflakes exhibit better electrochemical performances for supercapacitors and the ORR than Ni-MOF nanoflakes and ZIF-67.

\section{Conclusions}

We demonstrate an effective wet-chemical approach to achieve spherical hollow microstructures of assembled 2D $\mathrm{Ni} / \mathrm{Co}-\mathrm{MOF}$ nanoflakes and Ni-MOF nanoflakes. This approach leads to favorable $\mathrm{Ni} / \mathrm{Co}-\mathrm{MOF}$ nanoflake spherical microstructures with many exposed active sites. When applied in supercapacitors and the ORR, the $\mathrm{Ni} / \mathrm{Co}-$ MOF exhibits remarkable performances with a specific capacitance of $530.4 \mathrm{~F} \mathrm{~g}^{-1}$ at $0.5 \mathrm{~A} \mathrm{~g}^{-1}$ (higher than that of Ni-MOF (306.8 $\mathrm{F} \mathrm{g}^{-1}$ ) and ZIF-67 $\left(168.3 \mathrm{~F} \mathrm{~g}^{-1}\right)$ ), good rate capability, and robust cycling performance with no capacity fading after 2000 cycles. Besides, Ni/Co-MOF nanoflakes can be used as an advanced non-noble metal catalyst for the ORR with its excellent oxygen reduction catalytic activity, good durability, and good methanol tolerance. Taking their robust electrochemical performance in supercapacitors and ORR into account, our work provides a new concept to design and synthesize rationally tunable structures of 2D MOF nanoflakes to improve the electrochemical performance for energy storage and conversion.

Acknowledgements This work was financially supported by the National Natural Science Foundation of China (Nos. 21571157, U1604123, and 51473149), Outstanding Young Talent Research Fund of Zhengzhou University (1521320001), and the Open Project Foundation of Key Laboratory of Advanced Energy Materials Chemistry (Ministry of Education) (2017-29), Nankai University, and Open Project Foundation of Key Laboratory of Inorganic Synthesis and Preparation of Jilin University.

Open Access This article is distributed under the terms of the Creative Commons Attribution 4.0 International License (http://crea tivecommons.org/licenses/by/4.0/), which permits unrestricted use, distribution, and reproduction in any medium, provided you give appropriate credit to the original author(s) and the source, provide a link to the Creative Commons license, and indicate if changes were made.

\section{References}

1. R. Banerjee, H. Furukawa, D. Britt, C. Knobler, M. O'Keeffe, O.M. Yaghi, Control of pore size and functionality in isoreticular zeolitic imidazolate frameworks and their carbon dioxide selective capture properties. J. Am. Chem. Soc. 131(11), 3875-3877 (2009). doi:10.1021/ja809459e

2. A.U. Czaja, N. Trukhan, U. Muller, Industrial applications of metal-organic frameworks. Chem. Soc. Rev. 38(5), 1284-1293 (2009). doi: $10.1039 / \mathrm{b} 804680 \mathrm{~h}$

3. E. Jeong, W.R. Lee, D.W. Ryu, Y. Kim, W.J. Phang, E.K. Koh, C.S. Hong, Reversible structural transformation and selective gas adsorption in a unique aqua-bridged $\mathrm{Mn}$ (II) metal-organic framework. Chem. Commun. 49(23), 2329-2331 (2013). doi:10. 1039/c3ec00093a

4. T. Rodenas, I. Luz, G. Prieto, B. Seoane, H. Miro et al., Metalorganic framework nanosheets in polymer composite materials for gas separation. Nat. Mater. 14(1), 48-55 (2015). doi:10.1038/ nmat4113

5. N.L. Rosi, J. Eckert, M. Eddaoudi, D.T. Vodak, J. Kim, M. O'Keeffe, O.M. Yaghi, Hydrogen storage in microporous metalorganic frameworks. Science $\mathbf{3 0 0}(5622), 1127-1129$ (2003). doi: $10.1126 /$ science. 1083440

6. H. Wu, W. Zhou, T. Yildirim, Hydrogen storage in a prototypical zeolitic imidazolate framework-8. J. Am. Chem. Soc. 129(17), 5314-5315 (2007). doi:10.1021/ja0691932 
7. A. Morozan, F. Jaouen, Metal organic frameworks for electrochemical applications. Energy Environ. Sci. 5(11), 9269-9290 (2012). doi:10.1039/c2ee22989g

8. D. Wu, Z. Guo, X. Yin, Q. Pang, B. Tu, L. Zhang, Y.G. Wang, Q. $\mathrm{Li}$, Metal-organic frameworks as cathode materials for $\mathrm{Li}-\mathrm{O}_{2}$ batteries. Adv. Mater. 26(20), 3258-3262 (2014). doi:10.1002/ adma.201305492

9. S. Bai, X. Liu, K. Zhu, S. Wu, H. Zhou, Metal-organic framework-based separator for lithium-sulfur batteries. Nat. Energy 1(7), 16094 (2016). doi:10.1038/nenergy.2016.94

10. A. Mahmood, R. Zou, Q. Wang, W. Xia, H. Tabassum, B. Qiu, R. Zhao, Nanostructured electrode materials derived from metalorganic framework xerogels for high-energy-density asymmetric supercapacitor. ACS Appl. Mater. Interfaces 8(3), 2148-2157 (2016). doi:10.1021/acsami.5b10725

11. R.Q. Zou, H. Sakurai, Q. Xu, Preparation, adsorption properties, and catalytic activity of 3D porous metal-organic frameworks composed of cubic building blocks and alkali-metal ions. Angew. Chem. Int. Ed. 45(16), 2542-2546 (2006). doi:10.1002/anie. 200503923

12. R.Q. Zou, H. Sakurai, S. Han, R.Q. Zhong, Q. Xu, Probing the Lewis acid sites and $\mathrm{CO}$ catalytic oxidation activity of the porous metal-organic polymer $[\mathrm{Cu}(5$-methylisophthalate $)]$. J. Am. Chem. Soc. 129(27), 8402-8403 (2007). doi:10.1021/ja071662s

13. A. Corma, H. Garcia, F.X. Llabres, I. Xamena, Engineering metal organic frameworks for heterogeneous catalysis. Chem. Rev. 110(8), 4606-4655 (2010). doi:10.1021/cr9003924

14. M.A. Nasalevich, R. Becker, E.V. Ramos-Fernandez, S. Castellanos, S.L. Veber et al., Co@ $\mathrm{NH}_{2}-\mathrm{MIL}-125(\mathrm{Ti})$ : cobaloximederived metal-organic framework-based composite for light-driven $\mathrm{H}_{2}$ production. Energy Environ. Sci. 8(1), 364-375 (2015). doi: $10.1039 / \mathrm{c} 4 \mathrm{ee} 02853 \mathrm{~h}$

15. P.Z. Li, X.J. Wang, J. Liu, J.S. Lim, R. Zou, Y. Zhao, A triazolecontaining metal-organic framework as a highly effective and substrate size-dependent catalyst for $\mathrm{CO}_{2}$ conversion. J. Am. Chem. Soc. 138(7), 2142-2145 (2016). doi:10.1021/jacs.5b13335

16. R. Zou, P.Z. Li, Y.F. Zeng, J. Liu, R. Zhao et al., Bimetallic metal-organic frameworks: probing the lewis acid site for $\mathrm{CO}_{2}$ conversion. Small 12(17), 2334-2343 (2016). doi:10.1002/smll. 201503741

17. Z. Xu, L.L. Han, G.L. Zhuang, J. Bai, D. Sun, In situ construction of three anion-dependent $\mathrm{Cu}(\mathrm{I})$ coordination networks as promising heterogeneous catalysts for azide-alkyne "click" reactions. Inorg. Chem. 54(10), 4737-4743 (2015). doi:10.1021/ acs.inorgchem. 5 b00110

18. P. Horcajada, C. Serre, G. Maurin, N.A. Ramsahye, F. Balas et al., Flexible porous metal-organic frameworks for a controlled drug delivery. J. Am. Chem. Soc. 130(21), 6774-6780 (2008). doi:10.1021/ja710973k

19. K.M. Taylor-Pashow, J. Della Rocca, Z. Xie, S. Tran, W. Lin, Postsynthetic modifications of iron-carboxylate nanoscale metalorganic frameworks for imaging and drug delivery. J. Am. Chem. Soc. 131(40), 14261-14263 (2009). doi:10.1021/ja906198y

20. P. Horcajada, T. Chalati, C. Serre, B. Gillet, C. Sebrie et al., Porous metal-organic-framework nanoscale carriers as a potential platform for drug delivery and imaging. Nat. Mater. 9(2), 172-178 (2010). doi:10.1038/nmat2608

21. J. Gascon, A. Corma, F. Kapteijn, F.X. Llabrés i Xamena, Metal organic framework catalysis: quo vadis? ACS Catal. 4(2), 361-378 (2014). doi:10.1021/cs400959k

22. W. Xia, A. Mahmood, Z. Liang, R. Zou, S. Guo, Earth-abundant nanomaterials for oxygen reduction. Angew. Chem. Int. Ed. 55(8), 2650-2676 (2016). doi:10.1002/anie.201504830

23. H. Yu, D. Xu, Q. Xu, Dual template effect of supercritical $\mathrm{CO}_{2}$ in ionic liquid to fabricate a highly mesoporous cobalt metal- organic framework. Chem. Commun. 51(67), 13197-13200 (2015). doi:10.1039/c5cc04009d

24. S.L. Li, Q. Xu, Metal-organic frameworks as platforms for clean energy. Energy Environ. Sci. 6(6), 1656-1683 (2013). doi:10. 1039/c3ee40507a

25. Q.L. Zhu, W. Xia, T. Akita, R. Zou, Q. Xu, Metal-organic framework-derived honeycomb-like open porous nanostructures as precious-metal-free catalysts for highly efficient oxygen electroreduction. Adv. Mater. 28(30), 6391-6398 (2016). doi:10. 1002/adma.201600979

26. W. Xia, R.Q. Zou, L. An, D.G. Xia, S.J. Guo, A metal-organic framework route to in situ encapsulation of $\mathrm{Co}_{0} \mathrm{Co}_{3} \mathrm{O}_{4} @ \mathrm{C}$ core@bishell nanoparticles into a highly ordered porous carbon matrix for oxygen reduction. Energy Environ. Sci. 8(2), 568-576 (2015). doi:10.1039/c4ee02281e

27. S. Zhao, Y. Wang, J. Dong, C.-T. He, H. Yin et al., Ultrathin metal-organic framework nanosheets for electrocatalytic oxygen evolution. Nat. Energy 1, 16184 (2016). doi:10.1038/nenergy. 2016.184

28. A. Mahmood, W.H. Guo, H. Tabassum, R.Q. Zou, Metal-organic framework-based nanomaterials for electrocatalysis. Adv. Energy Mater. 6(17), 1600423 (2016). doi:10.1002/aenm.201600423

29. P. Pachfule, D. Shinde, M. Majumder, Q. Xu, Fabrication of carbon nanorods and graphene nanoribbons from a metal-organic framework. Nat. Chem. 8(7), 718-724 (2016). doi:10.1038/ nchem. 2515

30. W. Xia, A. Mahmood, R. Zou, Q. Xu, Metal-organic frameworks and their derived nanostructures for electrochemical energy storage and conversion. Energy Environ. Sci. 8(7), 1837-1866 (2015). doi:10.1039/c5ee00762c

31. J.R. Selman, Materials science. Poison-tolerant fuel cells. Science 326(5949), 52-53 (2009). doi:10.1126/science. 1180820

32. K.M. Choi, H.M. Jeong, J.H. Park, Y.B. Zhang, J.K. Kang, O.M. Yaghi, Supercapacitors of nanocrystalline metal-organic frameworks. ACS Nano 8(7), 7451-7457 (2014). doi:10.1021/ nn5027092

33. P. Zhou, Q. Xu, H. Li, Y. Wang, B. Yan, Y. Zhou, J. Chen, J. Zhang, K. Wang, Fabrication of two-dimensional lateral heterostructures of $\mathrm{WS}_{2} / \mathrm{WO}_{3} \mathrm{H}_{2} \mathrm{O}$ through selective oxidation of monolayer $\mathrm{WS}_{2}$. Angew. Chem. Int. Ed. 54(50), 15226-15230 (2015). doi:10.1002/anie.201508216

34. B. Zhang, F. Wang, C. Zhu, Q. Li, J. Song, M. Zheng, L. Ma, W. Shen, A facile self-assembly synthesis of hexagonal $\mathrm{ZnO}$ nanosheet films and their photoelectrochemical properties. Nano-Micro Lett. 8(2), 137-142 (2016). doi:10.1007/s40820-015-0068-y

35. X. Yang, K. Xu, R. Zou, J. Hu, A hybrid electrode of $\mathrm{Co}_{3-}$ $\mathrm{O}_{4} @ \mathrm{PPy}$ core/shell nanosheet arrays for high-performance supercapacitors. Nano-Micro Lett. 8(2), 143-150 (2016). doi:10. 1007/s40820-015-0069-x

36. A.K. Geim, K.S. Novoselov, The rise of graphene. Nat. Mater. 6(3), 183-191 (2007). doi:10.1038/nmat1849

37. F. Cao, M. Zhao, Y. Yu, B. Chen, Y. Huang et al., Synthesis of two-dimensional $\mathrm{CoS}_{1.097} / \mathrm{Nitrogen-doped} \mathrm{carbon} \mathrm{nanocompos-}$ ites using metal-organic framework nanosheets as precursors for supercapacitor application. J. Am. Chem. Soc. 138(22), 6924-6927 (2016). doi:10.1021/jacs.6b0254

38. S. Li, D. Wu, C. Cheng, J. Wang, F. Zhang, Y. Su, X. Feng, Polyaniline-coupled multifunctional 2D metal oxide/hydroxide graphene nanohybrids. Angew. Chem. Int. Ed. 52(46), 12105-12109 (2013). doi:10.1002/anie.201306871

39. S. Li, D. Wu, H. Liang, J. Wang, X. Zhuang, Y. Mai, Y. Su, X. Feng, Metal-nitrogen doping of mesoporous carbon/graphene nanosheets by self-templating for oxygen reduction electrocatalysts. ChemSusChem 7(11), 3002-3006 (2014). doi:10.1002/cssc. 201402680 
40. Q. Wang, R. Zou, W. Xia, J. Ma, B. Qiu et al., Facile synthesis of ultrasmall $\mathrm{CoS}_{2}$ nanoparticles within thin $\mathrm{N}$-doped porous carbon shell for high performance lithium-ion batteries. Small 11(21), 2511-2517 (2015). doi:10.1002/smll.201403579

41. J. Yang, F. Zhang, H. Lu, X. Hong, H. Jiang, Y. Wu, Y. Li, Hollow $\mathrm{Zn} / \mathrm{Co}$ ZIF particles derived from core-shell ZIF67@ZIF-8 as selective catalyst for the semi-hydrogenation of acetylene. Angew. Chem. Int. Ed. 54(37), 10889-10893 (2015). doi:10.1002/anie.201504242

42. H. Chen, L.F. Hu, M. Chen, Y. Yan, L.M. Wu, Nickel-cobalt layered double hydroxide nanosheets for high-performance supercapacitor electrode materials. Adv. Funct. Mater. 24(7), 934-942 (2014). doi:10.1002/adfm.201301747

43. R. Ma, J. Liang, K. Takada, T. Sasaki, Topochemical synthesis of $\mathrm{Co}-\mathrm{Fe}$ layered double hydroxides at varied $\mathrm{Fe} / \mathrm{Co}$ ratios: unique intercalation of triiodide and its profound effect. J. Am. Chem. Soc. 133(3), 613-620 (2011). doi:10.1021/ja1087216

44. N.S. McIntyre, M.G. Cook, X-ray photoelectron studies on some oxides and hydroxides of cobalt, nickel, and copper. Anal. Chem. 47(13), 2208-2213 (1975). doi:10.1021/ac60363a034

45. Y. Liu, J.A. Zhang, S.P. Wang, K.X. Wang, Z.M. Chen, Q. Xu, Facilely constructing $3 \mathrm{D}$ porous $\mathrm{NiCo}_{2} \mathrm{~S}_{4}$ nanonetworks for highperformance supercapacitors. New J. Chem. 38(9), 4045-4048 (2014). doi:10.1039/c4nj00816b

46. Q. Liu, J. Jin, J. Zhang, $\mathrm{NiCo}_{2} \mathrm{~S}_{4} @$ graphene as a bifunctional electrocatalyst for oxygen reduction and evolution reactions.
ACS Appl. Mater. Interfaces 5(11), 5002-5008 (2013). doi:10. 1021/am4007897

47. J. Tang, R.R. Salunkhe, J. Liu, N.L. Torad, M. Imura, S. Furukawa, Y. Yamauchi, Thermal conversion of core-shell metalorganic frameworks: a new method for selectively functionalized nanoporous hybrid carbon. J. Am. Chem. Soc. 137(4), 1572-1580 (2015). doi:10.1021/ja511539a

48. S. Zhong, C.X. Zhan, D.P. Cao, Zeolitic imidazolate frameworkderived nitrogen-doped porous carbons as high performance supercapacitor electrode materials. Carbon 85, 51-59 (2015). doi:10.1016/j.carbon.2014.12.064

49. N.L. Torad, R.R. Salunkhe, Y. Li, H. Hamoudi, M. Imura, Y. Sakka, C.C. Hu, Y. Yamauchi, Electric double-layer capacitors based on highly graphitized nanoporous carbons derived from ZIF-67. Chem. Eur. J. 20(26), 7895-7900 (2014). doi:10.1002/ chem.201400089

50. X. Deng, J. Li, S. Zhu, F. He, C. He, E. Liu, C. Shi, Q. Li, N. Zhao, Metal-organic frameworks-derived honeycomb-like $\mathrm{Co}_{3} \mathrm{O}_{4}$ /three-dimensional graphene networks/ $\mathrm{Ni}$ foam hybrid as a binder-free electrode for supercapacitors. J. Alloys Compd. 693, 16-24 (2017). doi:10.1016/j.jallcom.2016.09.096

51. M. Hamdani, R.N. Singh, P. Chartier, $\mathrm{Co}_{3} \mathrm{O}_{4}$ and Co-based spinel oxides bifunctional oxygen electrodes. Int. J. Electrochem. Sci. 5(4), 556-577 (2010) 